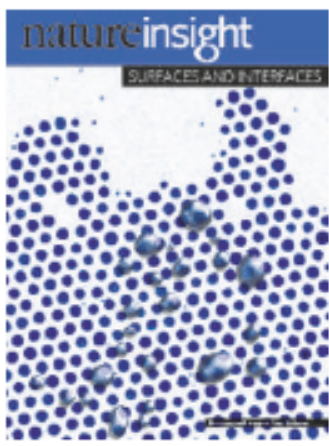

Cover illustration

Transmission electron microscopy image of a selfassembled superlattice of monodisperse colloidal cobalt nanocrystals synthesized under kinetic control in a hotorganic surfactant solution.

(Courtesy of Y. Yin and P.A. Alivisatos.) Water droplets have been added.

Editor, Nature
Philip Campbell
Insights Publisher
Sarah Greaves
Insights Editor
Lesley Anson
Production Editor
Maria Hodges
Senior Art Editor
Martin Harrison
Art Editor
Nik Spencer
Layouts
Clifford Saunders
Marie-Claire Patin
Sponsorship
Claire Hines
Claudia Banks
Production
Sue Gray
Marketing
Claire Aspinall
EditorialAssistant
Laura Shaw

\title{
SURFACES AND INTERFACES
}

\section{REVIEWS}

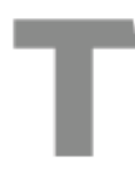

he importance of interfaces cannot be overestimated. They play a vital role in technological applications as diverse as catalysis, microelectronics, lubrication, corrosion, photography and in many environmental processes (including, infamously, stratospheric ozone destruction). Moreover, many of the biochemical reactions that sustain life occur at surfaces and interfaces.

Although their significance has been realized for centuries, surfaces and interfaces long evaded detailed scrutiny at the atomic scale. After all, they are not simply the final layer of, say, a piece of metal or liquid in contact with air, but an exceedingly thin region with properties distinct from those of the bulk material on either side. But the past 30 years have techniques that have delivered incisive insight into the composition and structure of a wide variety of surfaces and interfaces. As our understanding of these peculiar regions of matter grows, along with the range of characterization and manipulation tools at our disposal, we are in a position to use the unique environment of surfaces and interfaces to explore fascinating science and new applications.

This Insight aims to serve as a crystal ball, to give a flavour of the challenges and opportunities in our quest to fully understand surfaces and interfaces, and to precisely control their properties for applications that range from materials processing and information technology to biology and medicine. It goes almost without saying that the richness of the field, in terms of the systems and processes studied, the tools being used, and the diversity of applications that are being targeted, is superb. The choice of themes being reviewed here is thus inevitably somewhat eclectic, but we hope you enjoy the offering.

We are pleased to acknowledge the support of Unilever in producing this Insight. As always, Nature carries sole responsibility for all editorial content and peer review. seen the development of increasingly sophisticated
638 Aperspective onsurfaces and interfaces

D. L. Allara

640 Interfaces and the driving force of hydrophobic assembly

D. Chandler

648 Controlledmicrofluidic interfaces

J. Atencia \& D. J. Beebe

656 Polymer-supported membranes as models of the cell surface

M. Tanaka \& E. Sackmann

664 Colloidal nanocrystal synthesis and the organic-inor ganic interface Y. Yin \& A. P. Alivisatos

671 Engineering atomic and molecular nanostructures at surfaces

J. V. Barth, G. Costantini \& K. Kern 